\title{
Publisher's Note: The physics of the intergalactic medium [Rev. Mod. Phys. 81, 1405 (2009)]
}

\author{
Avery A. Meiksin \\ (Published 31 December 2009) \\ DOI: 10.1103/RevModPhys.81.1943 PACS number(s): 95.30.Dr, 95.35.+d, 98.62.Ra, 98.80.-k, 99.10.Fg
}

This paper was published online on 5 October 2009 with initial corrections that were made on 7 October 2009 and that subsequent to those corrections, new corrections were incorporated on 24 November 2009. The original corrections include a typographical error in Eq. (15). The third line of Eq. (15) should read as “...=1.100 $\times 10^{4} \ldots$. " The new corrections include an error in Eq. (89), as well as an error in the text on page 1441. Equation (89) should read as $d \mathbf{x}_{d} / d t=\mathbf{v}_{d}(\mathbf{x}, t) / a$. On page 1441, the eighth line of text under Eq. (100) should read as "...g is a Rayleigh random deviate in the range $0<g<\infty$ with unit mean...." The errors have been corrected as of 24 November 2009. The text is correct in the printed version of the journal. 